\title{
Going through or around the occlusion? All roads lead to Rome
}

\author{
Niccolò Ciardetti, Alessio Mattesini, Carlo Di Mario \\ Structural Interventional Cardiology, Department of Clinical and Experimental Medicine, \\ Careggi University Hospital, Florence, Italy
}

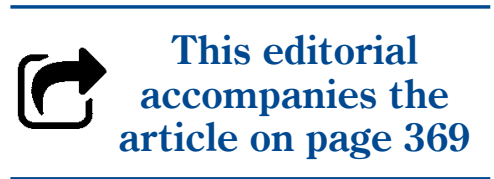

This meta-analysis approaches one of the most heavily debated aspects of the chronic total occlusion (CTO) technique. How soon should operators switch from the conventional wire escalation (WE)
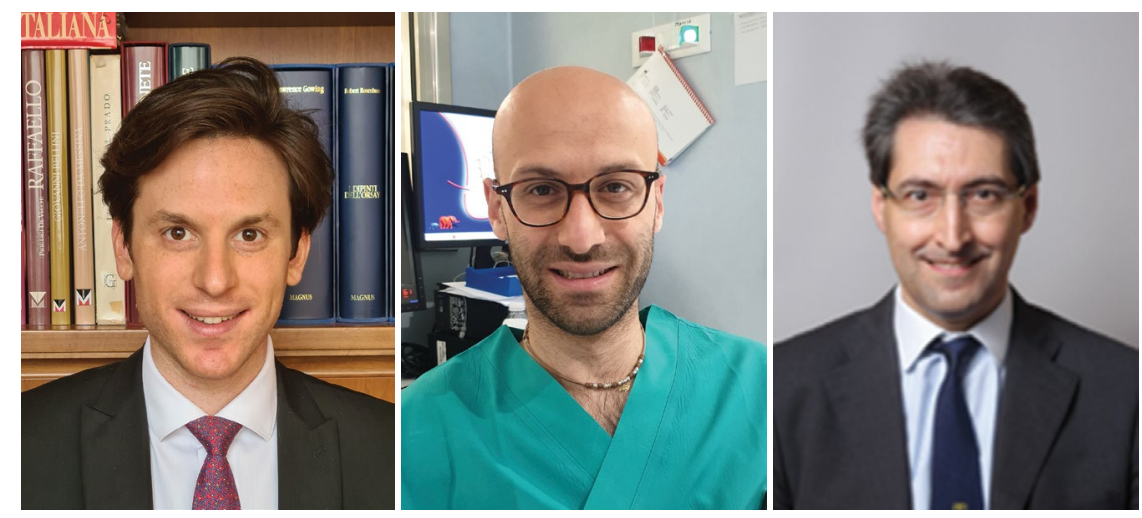

technique to the creation of a subintimal path. Peripheral interventionalists use the latter technique as standard in very long occlusions of the superficial femoral artery or infrapopliteal vessels but their reocclusion rate is high and stenting is used more rarely. Flow rather than lumen size appears to be the determinant of late patency in this setting.

For coronary interventions, the technique is very different and the adjunctive use of a partial or complete subintimal path remains very controversial. Progress in wire steerability within the occlusion and dedicated wires able to pierce calcified segments stepping down to softer wires in the remaining part of the procedure [1]. The same considerations can be repeated for retrograde recanalization with retrograde WE using the novel Gaia family (Asahi Intecc, Japan) of wires, often sufficient to regain the proximal end of the occlusion.

The question comes when the proximal or distal stump is ambiguous or when the length and tortuosity of the occluded segment makes safe handling of the CTO wires impossible, with high risk of wire exits. The ambiguity of the stump can occasionally be solved with intravascular ultrasound (IVUS) or pre-interventional coronary computed tomography-angiography but remains a driver to rely on the ability of a knuckled wire to follow the vessel architecture minimizing the risk of perforation [2]. The difficulty is hardly ever the ability to quickly gain ground within the occluded segment. Occasionally the knuckled wire can follow side-branches rather than the main vessel but this is not as frequent as with stiff devices such as the CrossBoss (Boston Scientific, USA). Most of the time, the problem comes at the end (proximal or distal) when reentry in the true lumen can become nearly impossible. Retrogradely, there is low pressure building up within the false lumen and the anterograde wire and balloon advanced can facilitate reentry with a traditional or modified reverse controlled anterograde retrograde tracking (CART) technique. Anterogradely, the situation is trickier. The use of a guide extension or deep intubation to reduce anterograde pressure strictly avoiding

Address for correspondence: Professor Carlo Di Mario, Department of Clinical and Experimental Medicine, Clinica Medica, Room 124, Careggi University Hospital, Largo Brambilla 3, 50139, Florence, Italy, tel/fax: +39 3486522399 , e-mail: carlo.dimario@unifi.it

This article is available in open access under Creative Common Attribution-Non-Commercial-No Derivatives 4.0 International (CC BY-NC-ND 4.0) license, allowing to download articles and share them with others as long as they credit the authors and the publisher, but without permission to change them in any way or use them commercially. 
contrast or flush may limit the size of the distal dissection and avoid full collapse of the distal lumen but we still miss a consistent reliable method to achieve successful wire dissection reentry. The only small randomized trial in this field compared anterograde dissection and reentry (ADR) with CrossBoss/StingRay and anterograde WE (AWE) and showed a similar procedure duration but the percentage of stand-alone ADR used as primary approach remained low (77\%) [3]. Large registries (RECHARGE; US Registry; UK Hybrid CTO Registry) with experienced ADR operators reported a $60-81 \%$ success rate [4-6]. Dual lumen catheters are more deliverable and may offer an alternative to StingRay but they miss the dedicated features that facilitate distal reentry with the StingRay balloon and should be considered more as a modified AWE technique with the use of parallel wires. Alternatives are scarce because IVUS guidance of distal reentry, with the exception of large less calcified vessels in the hands of very experienced IVUS operators, remains unpredictable. Pushing a wire to the distal vasculature (STAR) should be considered only a bail-out technique when all other options have failed and more of an investment procedure than a true procedural success, irrespective of the final flow achieved. In smaller vessels, for instance diagonals with ostial irretrievable dissections, mini-STAR is a viable option but this should not enter into the true CTO options.

The struggle between wire "manipulators" and wire "pushers" is more theoretical than real. The complexity of the lesion determines the technique and knuckling remains indispensable for very long calcified and tortuous occlusions. The difference is more in the time allowed to pass before switching strategy but this is also an important procedural step and the data presented in this article may favor a more liberal or more conservative use of dissection and reentry (DR) techniques based on long-term outcome.

There is evidence that the immediate outcome when techniques more complex than AWE are required is worse, with damage to collateral vessels as the main driver of adverse events for retrograde procedures and inability to protect and save branches distal to the occlusion as a driver of ADR complications [7]. The article by Zhao et al. [8] goes beyond the immediate procedural outcome to explore in 5265 patients the clinical results at 12-24 months, in terms of restenosis and adverse clinical events.

Analyzing 12 non-randomized cohort studies, the authors found that DR techniques, compared to
WE, are associated with higher risk of myocardial infarction (MI), target-vessel revascularization (TVR), in-stent restenosis (ISR), and in-stent reocclusion. Cardiovascular death and in-stent thrombosis were similar in the two groups.

Patients treated with a creation of a subintimal path required a higher number of stents and a greater mean stent length, in the face of more complex CTO lesions, as shown by significantly higher J-CTO score and longer CTO length. This could be a relevant limitation, since typically DR techniques are needed when CTO lesions are severely tortuous or calcified and WE is not sufficient for complete recanalization of the occluded segment.

Fortunately, the authors went even further: they performed subgroup analyses according to different approaches (anterograde or retrograde) and different DR techniques. They distinguished between patients treated with 'extensive' DR techniques - including CTO lesions recanalized with the STAR technique, mini-STAR, contrast-guided STAR and limited antegrade subintimal tracking - and those in whom 'limited' DR techniques were used - reverse-CART and device-facilitated techniques with the CrossBoss/ /StingRay system.

Long term endpoints were different based on DR techniques used: 'extensive' DR techniques have higher risk of TVR, ISR and composite endpoint (death, MI, TVR) compared to WE (Fig. 1A), while no differences were found between 'limited' DR and WE techniques (Fig. 1B), as well as between anterograde and retrograde approaches.

It seems clear that the issue is not the presence of a subintimal path but rather of extensive and uncontrolled dissections. Unfortunately, CTO lesion complexity was not consistently reported in the two DR groups, hence, it is not known if the higher risk is carried by the extensive dissection or by the intrinsic complexity of the disease. These clinical long-term data support the results of two smaller registries (ISAR-CTO and CONSISTENT-CTO) that performed follow-up angiography in all CTO patients, completing an assessment with optical coherence tomography $[9,10]$. Results were slightly different. CONSISTENT-CTO [9] performed 175 optical coherence tomography late after CTO recanalization, with patients divided nearly equally into DR techniques and no DR group. More than $90 \%$ of stent struts were covered in both groups with no features (frequency and length of uncovered strut segment) shown to be at risk for stent 

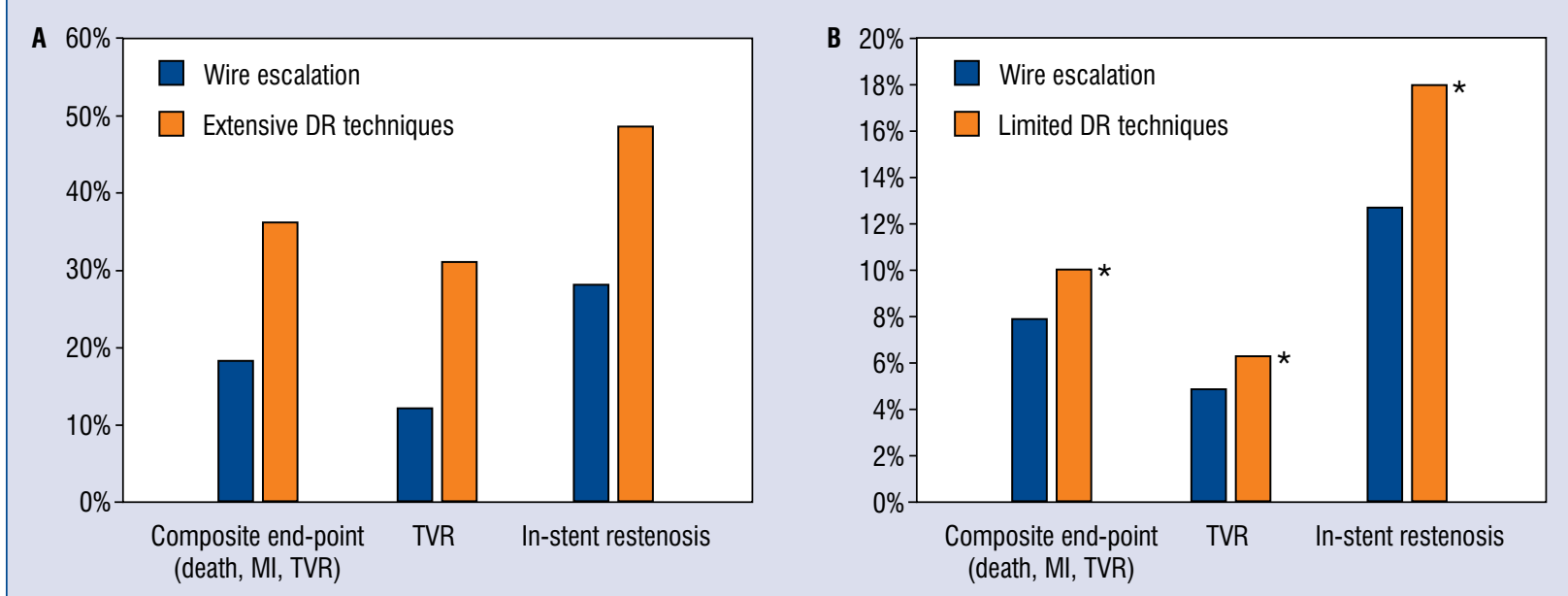

Figure 1. Long term endpoints according to the techniques used for recanalization; A. Wire escalation versus 'extensive' dissection and reentry (DR) techniques; B. Wire escalation versus 'limited' DR techniques; *Differences are not statistically significant; $\mathrm{MI}$ - myocardial infarction; TVR — target vessel revascularization.

thrombosis. ISAR-CTO [10], in a smaller number of patients and without the benefit of a consistent IVUS confirmation of the presence of a subintimal path during recanalization, showed a numerically higher percentage of uncovered struts and significantly more malapposed struts in the DR technique group. ISAR-CTO also showed rare cases of large aneurysms originating at the site of implantation of subintimal stents, confirming anectodical previous case reports.

After these studies and the reports summarized by Zhao et al. [8], the bitter controversies at times dominating the CTO sessions of the main interventional congresses can come to rest. Operators' experience and anatomy rather than aprioristic credos should drive individual technical decisions with no need for the CTO operators to choose one camp or the other.

\section{Conflict of interest: None declared}

\section{References}

1. Galassi AR, Werner GS, Boukhris M, et al. Percutaneous recanalisation of chronic total occlusions: 2019 consensus document from the EuroCTO Club. EuroIntervention. 2019; 15(2): 198-208, doi: 10.4244/EIJ-D-18-00826, indexed in Pubmed: 30636678.

2. Di Mario C, Mattesini A. What do you need for chronic total occlusion recanalization: swiftness or skillfulness? JACC Cardiovasc Interv. 2019; 12(6): 556-557, doi: 10.1016/j.jcin.2019.01.214, indexed in Pubmed: 30898252.

3. Karacsonyi J, Tajti P, Rangan BV, et al. Randomized comparison of a CrossBoss first versus standard wire escalation strategy for crossing coronary chronic total occlusions: the CrossBoss first trial. JACC Cardiovasc Interv. 2018; 11(3): 225-233, doi: 10.1016/j.jcin.2017.10.023, indexed in Pubmed: 29413236.

4. Maeremans J, Walsh S, Knaapen P, et al. The hybrid algorithm for treating chronic total occlusions in europe: the RECHARGE registry. J Am Coll Cardiol. 2016; 68(18): 1958-1970, doi: 10.1016/j. jacc.2016.08.034, indexed in Pubmed: 27788851.

5. Christopoulos G, Karmpaliotis D, Alaswad K, et al. Application and outcomes of a hybrid approach to chronic total occlusion percutaneous coronary intervention in a contemporary multicenter US registry. Int J Cardiol. 2015; 198: 222-228, doi: 10.1016/j. ijcard.2015.06.093.

6. Wilson WM, Walsh SJ, Yan AT, et al. Hybrid approach improves success of chronic total occlusion angioplasty. Heart. 2016; 102(18): 1486-1493, doi: 10.1136/heartjnl-2015-308891, indexed in Pubmed: 27164918.

7. Song L, Maehara A, Finn MT, et al. Intravascular ultrasound analysis of intraplaque versus subintimal tracking in percutaneous intervention for coronary chronic total occlusions and association with procedural outcomes. JACC Cardiovasc Interv. 2017; 10(10): 1011-1021, doi: 10.1016/j.jcin.2017.02.043, indexed in Pubmed: 28521919.

8. Zhao Y, Peng H, Li X, et al. The impact of dissection and re-entry versus wire escalation techniques on long-term clinical outcomes in patients with chronic total occlusion lesions following percutaneous coronary intervention: An updated meta-analysis. Cardiol J. 2021; 28(3): 369-383, doi: 10.5603/CJ.a2020.0026, indexed in Pubmed: 32104900.

9. Walsh SJ, Hanratty CG, McEntegart M, et al. Intravascular healing is not affected by approaches in contemporary CTO PCI: the CONSISTENT CTO study. JACC Cardiovasc Interv. 2020; 13(12): 1448-1457, doi: 10.1016/j.jcin.2020.03.032, indexed in Pubmed: 32553333.

10. Xhepa E, Cassese S, Rroku A, et al. Subintimal versus intraplaque recanalization of coronary chronic total occlusions: mid-term angiographic and OCT findings from the ISAR-OCT-CTO registry. JACC Cardiovasc Interv. 2019; 12(19): 1889-1898, doi: 10.1016/j.jcin.2019.04.049, indexed in Pubmed: 31521651. 